\title{
Pragmatic Distributed Algorithm for Spectral Access in Cooperative Cognitive Radio Networks
}

\author{
Wei Liang, Soon Xin Ng, Jiao Feng, and Lajos Hanzo
}

\begin{abstract}
A pragmatic distributed algorithm (PDA) is proposed for supporting the efficient spectral access of multiple Primary Users (PUs) and Cognitive Users (CUs) in cooperative Cognitive Radio (CR) networks. The novelty of our PDA is that the PUs negotiate with the CUs concerning the specific amount of relaying and transmission time, the $\mathrm{CU}$ is granted, which the $\mathrm{CU}$ will either accept or decline. The CUs may serve as relay nodes for relaying the signal received from the PUs to their destinations, while both the PUs' and the CUs' minimum rate requirements are satisfied. This will reduce the required transmission power and/or increase the transmission rate of the PU. Our results show that the proposed scheme performs better than the benchmarker, despite its significantly lower overhead and complexity. Moreover, we show that the cooperative spectral access based on our PDA reaches an equilibrium, when it is repeated for a sufficiently long duration. These benefits are achieved, because the PUs are motivated to cooperate by the incentive of achieving a higher PU rate, whilst non-cooperation can be discouraged with the aid of a limited-duration punishment. Furthermore, we invoke an attractive practical adaptive Turbo Trellis Coded Modulation (ATTCM) scheme, which appropriately adjusts the code rate and the modulation mode according to the near-instantaneous channel conditions. It was found that the joint design of coding, modulation and user-cooperation may lead to significant mutual benefits for all the PUs and the CUs.
\end{abstract}

Index Terms-Cognitive radio network, cooperative communication, spectrum access strategy, matching algorithm, ATTCM.

\section{INTRODUCTION}

$\mathbf{C}$ OGNITIVE radio (CR) was found to be able to improve the spectral efficiency by exploiting the available spectrum holes [1]-[3]. If the spectrum is not used by the Primary Users (PUs), then the Cognitive Users (CUs) have the opportunity to access it for their secondary communications based on the CR technique. According to the CR protocol, the device listens to the received signal and identifies the spectrum holes, using either time or frequency domain sensing [1][3]. In this context, the most common paradigms associated with CRs are the so-called underlay, overlay and interweave networks [4].

Manuscript received May 3, 2013; revised September 30, 2013 and February 13,2014 . The editor coordinating the review of this paper and approving it for publication was M. Tao.

The financial support of the European Research Council's Advanced Fellow Grant, that of the India-UK Advanced Technology Centre, as well as that of the European Union's Seventh Framework Programme (FP7/2007-2013) under the auspices of the CONCERTO project (grant agreement no 288502) is gratefully acknowledged. We would also like to express our gratitude to $\mathrm{Dr} \mathrm{S}$. Bayat and Dr Y. Li from the School of Electrical \& Information Engineering, University of Sydney, for the fruitful discussions on game theory.

The authors are with the School of ECS, University of Southampton, SO17 1BJ, UK (e-mail: \{w17g09, sxn, jf11g09, lh\}@ecs.soton.ac.uk).

Digital Object Identifier 10.1109/TCOMM.2014.030214.130326
Cooperative communication is a novel communication paradigm that promises significant capacity and multiplexing gain improvements in wireless networks and it is capable of supporting the users either at an improved integrity or throughput with the advent of user cooperation [5]-[7]. The most popular cooperative protocols are the Amplify-And-Forward (AAF) as well as the Decode-And-Forward (DAF) schemes. The benefits of employing cooperative communication in $\mathrm{CR}$ networks have been studied in [8]-[11]. In [8], a CU may act as a Relay Node (RN) for other CUs in the cooperation among the CU peers, which may have different available spectrum to the CU. Additionally, the cooperation between PUs and CUs, was studied in [9], where the PUs have a higher priority than the CUs. Thus cooperation would allow the PUs to transmit at a lower power and/or at a higher throughput, while at the same time enabling the CUs to communicate using the released spectrum. In our work, we consider a cooperative CR scheme, which relies on the cooperation of the source PU or PU transmitter $(\mathrm{Pt})$ and the destination PU or PU receiver $(\mathrm{Pr})$, where the CUs assist the PUs' transmission in exchange for spectral access. This is commonly referred to as the overlay paradigm, which was studied by considering a single PU in [10], [11]. However, in these scenarios the PU's improved performance does not necessarily translate into a satisfactory performance for the CUs. In some cases, the CUs have limited spectral access opportunities, if the PUs have their own data to transmit. Hence the CU has to wait for the PU to cease its transmissions [11]. Cooperation strategies involving multiple PUs were considered in [12]-[15].

Different PUs may operate using different licensed bands in the same geographical location. The authors of [15] considered multiple PUs and CUs. Moreover, all PUs and CUs may be considered to be selfish, hence they may only be concerned about their own benefit, pursuing their own best strategies for maximizing their own sum rate or throughput. A conventional distributed algorithm (CDA) was proposed in [12], which may be viewed as an evolution from the dynamic auction algorithms [16], [17], since it considers a resourceallocation [18] framework that facilitates a joint competitive strategy of the PUs and the CUs conceived for accessing the spectral resources. Moreover, the authors of [12] conceived a non-cooperative game, which employs the CDA for efficiently representing the interaction among the competing PUs, where each PU chooses its allocation independently of the others in order to improve its own performance. Explicitly, a spectral access strategy is designed for multiple PUs and CUs, where the PUs and CUs are carefully paired for ensuring that both the PUs' and the CUs' minimum sum-rate requirements are satisfied. Each paired CU assists in relaying its paired-PU's 
signal in exchange for a transmission opportunity using the PU's spectrum. However, the PUs under the CDA would sometimes compete among themselves for cooperating with the same relay, which may degrade both their utility and throughput. In contrast to the CDA, we proposed a protocol, which may be classified as a repeated game [19]-[21] where all PUs are capable of cooperating with each other. Thus they are motivated to form a grand coalition [22], [23] for achieving an increased expected PU rate by discouraging the PUs from competing with each other for the same CU's assistance. Furthermore, the concept of a penalty/punishment is introduced [19], which is imposed only for a carefully selected finite period for the sake of discouraging non-cooperation. Finally, we extended the PDA based repeated game concept to practical systems employing realistic coding and modulation schemes.

In $\mathrm{CR}$ systems the link-quality varies across a wide range, which cannot be efficiently mitigated by using power-control. Hence adaptive coded modulation [24] is proposed for accommodating these differences. Initially we have considered a pair of idealistic adaptive schemes based on both the Continuous-input Continuous-output Memoryless Channel (CCMC) and the Discrete-input Continuous-output Memoryless Channel (DCMC) [25]. More specifically, the CCMC based adaptive scheme assumes that idealistic coding and modulation schemes are employed for communicating exactly at Shannon's capacity. By contrast, the DCMC based adaptive scheme assumes that an idealistic capacity-achieving code is employed for allowing the PSK/QAM modulation schemes considered to operate right at the modulation-dependent DCMC capacity. In contrast to the idealized CCMC and DCMC schemes, we also make use of a range of practical power- and bandwidth-efficient Turbo Trellis Coded Modulation (TTCM) schemes ${ }^{1}$ in our CR system. Explicitly, TTCM is a joint coding and modulation scheme that has a structure similar to binary turbo codes, where two identical parallelconcatenated Trellis Coded Modulation [26] schemes are employed as component codes. The transmission rate/throughput of our system is adapted according to the instantaneous channel conditions. A more vulnerable, but higher-throughput TTCM scheme is employed when the channel conditions are good, while a lower-throughput but more robust TTCM scheme is used, when the channel conditions are poor.

The contribution of this paper is twofold. First, we proposed the PDA concept for efficient spectrum access, which is invoked for cooperation amongst the PUs for obtaining an improved performance for the PUs. Additionally, this PDA is formulated as a punishment based repeated game for attaining equilibrium. Secondly, we extended the repeated game concept to both the idealized perfect capacity-achieving coding scheme and to an attractive practical adaptive coded modulation scheme.

The rest of the paper is organized as follows. The system model of the cooperative CR network considered is outlined in Section II, while our spectral access strategy is detailed in Section III. Our realistic spectral access strategy based

\footnotetext{
${ }^{1}$ Other coding and modulation scheme can also be employed in our $\mathrm{CR}$ framework.
}

TABLE I

THE MAIN NOTATIONS OF OUR SYSTEM

\begin{tabular}{|c|c|}
\hline Notations & Description \\
\hline $\begin{array}{l}L_{P U} \text { or } \\
L\end{array}$ & Number of PUs \\
\hline $\begin{array}{l}L_{C U} \text { or } \\
K\end{array}$ & Number of CUs \\
\hline$l$ & Index of PUs \\
\hline$k$ & Index of CUs \\
\hline$R_{l, r e q}^{P U}$ & Rate requirement of PU \\
\hline$R_{k, r e q}^{C U}$ & Rate requirement of $\mathrm{CU}$ \\
\hline$\beta_{l, k}$ & Time allocation fraction \\
\hline$\tau$ & Step size of time allocation fraction \\
\hline$\gamma_{P U}$ & Transmit SNR of PU \\
\hline$\gamma_{C U}$ & Transmit SNR of CU \\
\hline$\epsilon_{l, k}$ & Time fraction \\
\hline$m_{l, k}$ & Matching matrix entry \\
\hline$\alpha$ & Path-loss exponent \\
\hline$\varrho$ & Pathloss \\
\hline$P_{\text {match }}$ & Relative frequency of successfully matched PUs \\
\hline
\end{tabular}

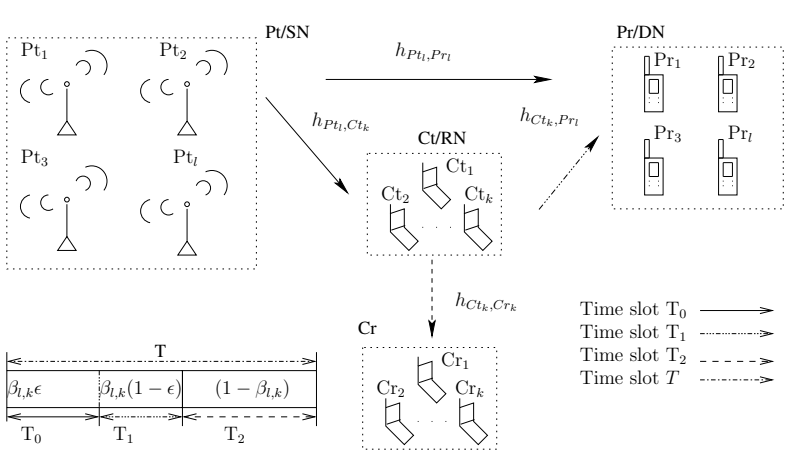

Fig. 1. The spectrum-access model for primary user and cognitive user.

on an adaptive coded modulation scheme is described in Section V. The performance of the proposed schemes is evaluated in Section VI. Finally, our conclusions are presented in Section VII.

\section{SySTEM MODEL}

In line with [12], we consider an overlay cooperative CR scheme comprising $L_{P U}$ number of "PU transmitter $(\mathrm{Pt})$ and PU receiver (Pr) pairs"2, namely ( $\left.\left\{P t_{l}\right\}_{l=1}^{L_{P U}},\left\{P r_{l}\right\}_{l=1}^{L_{P U}}\right)$, with the $l$ th pair having a rate requirement of $R_{P U_{l}, r e q}$, and with each pair occupying a unique spectral band of a constant width. In our scheme, there are $L_{C U}$ number of "CU transmitter $(\mathrm{Ct})$ and $\mathrm{CU}$ receiver $(\mathrm{Cr})$ pairs"3, namely ( $\left\{C t_{k}\right\}_{k=1}^{L_{C U}},\left\{C r_{k}\right\}_{k=1}^{L_{C U}}$ ) pairs, with the $k$ th pair having a requirement of $R_{C U_{k}, r e q}$, and seeking to obtain access to a spectral band occupied by a (Pt, Pr) pair. Each Pt attempts to grant spectral access to a unique $(\mathrm{Ct}, \mathrm{Cr})$ pair in exchange for the $\mathrm{Ct}$ cooperatively relaying the Pt's data to the corresponding Pr. Four types of matching algorithms will be described in Section III. Our main notations are shown in Table I.

Fig. 1 illustrates the time period allocation of the PUs and CUs, where $T$ is the original time period allocated for

${ }^{2}$ The Pt-Pr pair constitutes the PU's transceiver. We represent the PU acting as the source node by Pt. Similarly, Pr denotes the PU which acts as the destination node PU.

${ }^{3}$ The Ct-Cr pair is the CU's transceiver. The notation $\mathrm{Ct} / \mathrm{RN}$ represents the $\mathrm{CU}$ that acts as the relay node for helping the PU's transmission, while $\mathrm{Ct}$ denotes the $\mathrm{CU}$ that act as the source node $(\mathrm{SN})$. Similarly, the $\mathrm{Cr}$ denotes the destination node of a $\mathrm{Ct}$. 
the Pt to transmit its source message to the Pr. We will refer to $\left\{\beta_{l, k}\right\}_{l=1 k=1}^{L_{P U} L_{C U}}$ as the time allocation fraction, where $0<\beta_{l, k}<1$. When the $\mathrm{Pt}$ is assisted by a $\mathrm{Ct} / \mathrm{RN}$, the Pt relies on a time-fraction of $\beta_{l, k} T$ to convey the source message to the $\mathrm{Pr}$ and $\mathrm{Ct} / \mathrm{RN}$. More specifically, the Pt simultaneously transmits its message to $\mathrm{Pr}$ and $\mathrm{Ct} / \mathrm{RN}$ during the $T_{0}=\beta_{l, k} T \epsilon_{l, k}$ time-period, where $0<\epsilon_{l, k}<1$. Additionally, the $\mathrm{Ct} / \mathrm{RN}$ cooperatively relays the Pt's signal to $\mathrm{Pr}$ in the subsequent $T_{1}=\beta_{l, k}\left(1-\epsilon_{l, k}\right) T$ time-periods. Then the $\operatorname{Pr}$ applies maximum ratio combining for detecting the signal received from the Pt during the first $T_{0}$ time period, and the signal received from the $\mathrm{Ct} / \mathrm{RN}$ in the subsequent $T_{1}$ time periods. After the PU has ceased its transmission, the system will allow the CUs to transmit their informations to the other CUs by using the remaining time period of $T_{2}=\left(1-\beta_{l, k}\right) T$ for their own communications. In other words, a $\mathrm{Ct} / \mathrm{RN}$ assists in saving some of the transmission powers of the Pt due to the reduction of the transmission period from $T$ to $\left(T_{0}+T_{1}\right)$. The Pt transmits during $T_{0}$, while the $\mathrm{Ct} / \mathrm{RN}$ forwards the source message during $T_{1}$ and the $\mathrm{Ct} / \mathrm{SN}$ can broadcast its message to other CUs during the time period $T_{2}$. Let us assume that the transmission power per unit frequency transmitted from the Pt is $P_{S}$ watts/Hz and the target transmission rate is $R_{P U}$ bits/s.

During the first time slot (TS) $T_{0}$, the PU broadcasts the source message $x$ to both the $\mathrm{Pr}$ and to the $\mathrm{Ct} / \mathrm{RN}$. The signal received at the $\operatorname{Pr}$ is given by:

$$
y_{P t_{l}, P r_{l}}=\sqrt{P_{S}} h_{P t_{l}, P r_{l}} x+n_{P t_{l}, P r_{l}},
$$

and the signal received at the $\mathrm{Ct} / \mathrm{RN}$ is:

$$
y_{P t_{l}, C t_{k}}=\sqrt{P_{S}} h_{P t_{l}, C t_{k}} x+n_{P t_{l}, C t_{k}} .
$$

During the second TS $T_{1}$ the $\mathrm{Ct} / \mathrm{RN}$ would forward the source message to the Pr using the transmission power of $P_{C R}$ watts/Hz. Similarly, the signal received by the Pr under the $\mathrm{AAF}$ protocol via the RD link may be expressed as:

$$
y_{C t_{k}, P r_{l}}=\omega_{A} \sqrt{P_{C R}} h_{C t_{k}, P r_{l}} y_{P t_{l}, C t_{k}}+n_{C t_{k}, P r_{l}},(3)
$$

where $\omega_{A}=\frac{1}{\sqrt{P_{S}\left|h_{P t_{l}, C t_{k}}\right|^{2}+N_{0}}}$ [27] is the amplification factor, while $n_{P t_{l}, P r_{l}}, n_{P t_{l}, C t_{k}}$ and $n_{C t_{k}, P r_{l}}$ are the Gaussian noise vectors, which have a zero mean and a noise variance of $N_{0} / 2$ per dimension. The channel gain terms $h_{P t_{l}, P r_{l}}, h_{P t_{l}, C t_{k}}$ and $h_{C t_{k}, P r_{l}}$ are assumed to be Rayleigh distributed, obeying the complex-valued Gaussian distribution of $\mathcal{C N}(0,1)$. In our system, the path loss is included in the channel gain term. In our scheme, both the $\mathrm{Pt}$ and the $\mathrm{Ct} / \mathrm{RN}$ utilize the same bandwidth. The achievable instantaneous rate of the $l$ th PU when employing the $k$ th $\mathrm{CU}$ at a given $\beta_{l, k}$ may be represented as:

$$
R_{l, k}^{P U}\left(\beta_{l, k}\right)=C_{P U_{l, k}} \beta_{l, k} .
$$

where the capacity of PU $C_{P U_{l, k}}$ based on the Shannonian theory is given by:

$$
C_{P U_{l, k}}=\frac{T}{2} \log _{2}\left[1+\frac{\gamma_{P U}\left|h_{P t_{l}, P r_{l}}\right|^{2}}{d_{P t_{l}, P r_{l}}^{\alpha}}+f_{P t, C t, P r}\right],
$$

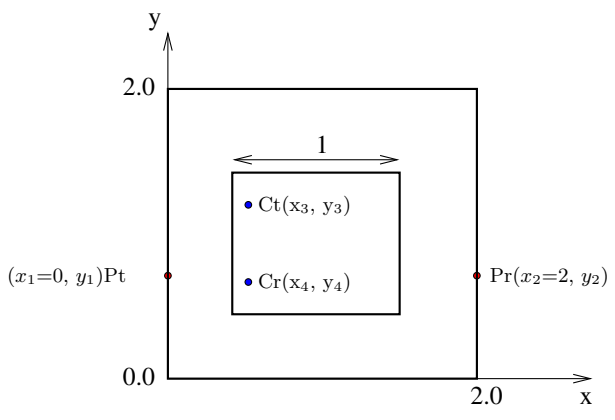

Fig. 2. The system design of distance setting.

where we have

$f_{P t, C t, P r}=$

$\frac{\gamma_{P U} \gamma_{C U}\left|h_{P t_{l}, C t_{k}}\right|^{2}\left|h_{C t_{k}, P r_{l}}\right|^{2}}{\gamma_{P U}\left|h_{P t_{l}, C t_{k}}\right|^{2} d_{C t_{l}, P r_{l}}^{\alpha}+\gamma_{C U}\left|h_{C t_{k}, P r_{l}}\right|^{2} d_{P t_{l}, C t_{l}}^{\alpha}+d_{P t_{l}, C t_{l}}^{\alpha} d_{C t_{l}, P r_{l}}^{\alpha}}$

The factor $\frac{1}{2}$ in Eq. (5) is due to the time fraction $\epsilon_{l, k}=\frac{1}{2}$, when we have $T_{0}=T_{1}$, where the Pt utilizes the first TS $T_{0}$ and the $\mathrm{Ct} / \mathrm{RN}$ uses the second TS $T_{1}$ to transmit the PU's signals. If the $T_{0} \neq T_{1}$, then the rate $R_{l, k}^{P U}$ at a given $\beta_{l, k}$ of the cooperative relay channel is given by:

$$
R_{l, k}^{P U}=\beta_{l, k} \epsilon_{l, k} T \log _{2}\left[1+\frac{\gamma_{P U}\left|h_{P t_{l}, P r_{l}}\right|^{2}}{d_{P t_{l}, P r_{l}}^{\alpha}}+f_{P t, C t, P r}\right],
$$

Note that the transmit $\mathrm{SNR}^{4}$ of the PU is $\gamma_{P U}=\frac{P_{s}}{N_{0}}$ and that of the $\mathrm{CU}$ is $\gamma_{C U}=\frac{P_{C R}}{N_{0}}$. Moreover, in the non-cooperative scenario, the achievable sum rate of the direct link between $\mathrm{Pt}_{l}$ and $\operatorname{Pr}_{l}$ is given by:

$$
C_{P U}^{*}=T \log _{2}\left[1+\frac{\gamma_{P U}\left|h_{P t_{l}, P r_{l}}\right|^{2}}{d_{P t_{l}, P r_{l}}^{\alpha}}\right],
$$

while the minimum rate requirement of the PU is given by $R_{l, r e q}^{P U}=C_{P U}^{*}$.

The achievable transmission rate of the $k$ th $\mathrm{CU}$ when assisting the $l$ th PU at a given $\beta_{l, k}$ is formulated as:

$$
R_{l, k}^{C U}\left(\beta_{l, k}\right)=\left(1-\beta_{l, k}\right) T \log _{2}\left[1+\gamma_{C U}\left|h_{C t_{k}, C r_{k}}^{(l)}\right|^{2}\right]
$$

where the channel $h_{C t_{k}, C r_{k}}^{(l)}$ depends on the frequency band provided by $C t_{l}$, while the pathloss is $\varrho=1 / d_{a b}^{\alpha}$ [27] and $d_{a b}$ is the geometrical distance between node $a$ and node $b$, while the path-loss exponent considered in our simulations is $\alpha=4$. Moreover, we assume that Pt and Pr are located at the opposite sides of a square at a normalized distance of two, thus $d_{\mathrm{Pt}_{l}, \mathrm{Pr}_{l}}=2.0$ as considered in [12] . Additionally, the $\mathrm{Cts} / \mathrm{RN}$ and $\mathrm{Crs}$ are assumed to be randomly located within an internal square having an edge-length of one, hence we have $\left(0<d_{C t_{k}, C r_{k}}<\sqrt{2}\right)$. As shown in Fig. 2, the distance between $\mathrm{Pt}$ and $\mathrm{Ct} / \mathrm{RN}$ is defined as

$$
d_{P t_{l}, C t_{k}}=\sqrt{\left|x_{3}-x_{1}\right|^{2}+\left|y_{3}-y_{1}\right|^{2}},
$$

\footnotetext{
${ }^{4}$ The definition of the transmit SNR is unconventional, because it relates the transmit-power to the noise-power at the receiver, which are quantities measured at different locations. Nonetheless, this convenient definition simplifies our discussion, as proposed in [28].
} 
while the distance between $\mathrm{Ct} / \mathrm{RN}$ and $\mathrm{Pr}$ is given by:

$$
d_{C t_{k}, P r_{l}}=\sqrt{\left|x_{2}-x_{3}\right|^{2}+\left|y_{2}-y_{3}\right|^{2}},
$$

where $x_{1}=0.0, x_{2}=2.0$ and $0 \leq y_{2}=y_{1} \leq 2$. Furthermore, we consider an outdoor environment, where the path-loss exponent [29] is given by $\alpha=4$.

\section{The Matching Algorithm}

In this section, we briefly highlight the matching algorithm [12] invoked for determining the spectral access for each $(\mathrm{Pt}, \mathrm{Pr})$ and $(\mathrm{Ct}, \mathrm{Cr})$ pairs.

\section{A. Preference Lists}

Before any offer is made to the CUs, the PUs construct a preferred list of CUs, which can satisfy the PU's rate requirement. Specifically, each $\mathrm{Pt}$ has a preference list of $\mathrm{Cts} / \mathrm{RN}$ that may assist in relaying its message, so that its achievable sum rate becomes higher than its minimum sumrate requirement. Thus, the preference list for $\mathrm{Pt}_{l}$ is given by:

$$
P U L I S T_{l}=\left\{\left(C t_{\kappa(k)}, C r_{\kappa(k)}\right)\right\}_{k=1}^{L_{C U}},
$$

where the function $\kappa(k)$ satisfies the following conditions:

$$
R_{l, \kappa(k)}^{P U}\left(\beta_{l, \kappa(k)}\right)>R_{l, r e q}^{P U}, k \in\left(1, \ldots, L_{C U}\right) .
$$

The index of the CUs may be recorded in the PULIST, while their corresponding rate has satisfied the PU's rate requirement. Additionally, we have assumed that the first $\mathrm{Ct}_{\kappa}(k)$ at the top of the PULIST $T_{l}$ provides the highest rate $R_{l, \kappa(k)}^{P U}\left(\beta_{l, \kappa(k)}\right)$. Similarly, each CU also has its preferred PU list, and if it transmits in the spectral band occupied by the preferred PUs then its achievable transmission rate is higher than its minimum sum-rate requirement, $R_{k, r e q}^{C U}$. Thus, the preference list for $\mathrm{Ct}_{k}$ is given by:

$$
C U L I S T_{k}=\left\{\left(P t_{\iota(l)}, \operatorname{Pr}_{\iota(l)}\right)\right\}_{l=1}^{L_{P U}},
$$

where the function $\iota(l)$ satisfies the following conditions:

$$
R_{\iota(l), k}^{C U}\left(\beta_{\iota(l), k}\right)>R_{k, r e q}^{C U}, l \in\left(1, \ldots, L_{P U}\right) .
$$

Again, the ordering of the $\mathrm{CULIST}_{k}$ also range from the highest to the lowest.

\section{B. Algorithm of Spectrum Access for our scheme}

1) Centralized Algorithm (CA): In the centralized algorithm (CA), we consider all possible matching of the $(\mathrm{Pt}, \mathrm{Pr})$ and $(\mathrm{Ct}, \mathrm{Cr})$ pairs, and then select that particular matched pair, which has the maximum sum rate. In our system, we aim for ensuring that each PU pair and CU pair has satisfied its minimum rate requirement. Here, both PUs and CUs are selfish if they choose a strategy that maximize their own utility, e.g. sum rate. This optimization cannot be achieved simultaneously for all PUs and CUs, since having a higher utility for the PU will result in a lower utility for the matched $\mathrm{CU}$, and vice-versa. By definition, in our cooperative $\mathrm{CR}$ scheme we give higher priority to the PUs. In particular, we focus our attention on maximizing the PUs' utility. We define a $\left(L_{P U} \times L_{C U}\right)$-element matching matrix $M$, where the $l$ th $\mathrm{PU}$ is matched to the $k$ th $\mathrm{CU}$, if their corresponding matching matrix entry is given by $m_{l, k}=1$ and $m_{l, k}=0$ otherwise. We also define a $\left(L_{P U} \times L_{C U}\right)$-element time-slot allocation matrix $T$ with elements $\beta_{l, k}$. Then the optimization problem of considering matched PUs can be formulated as [14], [30]:

$$
\begin{aligned}
\left\{M^{c e n}, T^{c e n}\right\}= & \arg \max _{M, T} \sum_{l=1}^{L_{P U}} \sum_{k=1}^{L_{C U}} m_{l, k} R_{l, k}^{P U}\left(\beta_{l, k}\right), \\
\text { s.t. } \quad & (a) R_{l, k}^{P U}\left(\beta_{l, k}\right) \geq R_{l, r e q}^{P U}, \forall l \forall k \\
& (b) R_{l, k}^{C U}\left(\beta_{l, k}\right) \geq R_{k, r e q}^{C U}, \forall l \forall k \\
& (c) 0 \leq \beta_{l, k} \leq 1, \forall l \forall k \\
& (d) \sum_{l=1}^{L_{P U}} m_{l, k} \leq 1, \forall k \\
& \left(\text { e) } \sum_{k=1}^{L_{C U}} m_{l, k} \leq 1, \forall l .\right.
\end{aligned}
$$

The conditions (a) and (b) are stipulated for ensuring that the minimum rate requirement of the PUs and CUs can be achieved. Condition (c) ensures that the TS allocation is limited to its bound. Finally, conditions (d) and (e) are imposed for ensuring that each PU(CU) will only be matched to one $\mathrm{CU}(\mathrm{PU})$. We assume that each transmitter-receiver pair has the knowledge of the number of PUs and CUs in the network. Without loss of generality, we also assume that the number of CUs is higher than the number of PUs.

By referring to Eq. (16), it becomes plausible that the optimization problem is non-linear and requires an exhaustive search over all possible matching pairs and TS allocation combinations. If the PUs and CUs always want to maximize their own utilities, then the outcome of the optimization problem may not be in the best interests of at least one of those users, hence the centralized approach may not be ideal. When the optimization problem is solved, the resultant matching information relating the PUs and CUs to each other will have to be transmitted to the corresponding users. The amount of overhead required for this side-information increases with the number of users, which may become quite high, rendering it impractical. We may consider solving the optimization problem of maximizing the sum rate of matched CUs and the total sum rate of matched PUs and CUs in our future research.

2) Conventional Distributed Algorithm (CDA): The key idea of this algorithm is that each $(\mathrm{Pt}, \mathrm{Pr})$ pair trades with a particular $(\mathrm{Ct}, \mathrm{Cr})$ pair for the sake of attaining mutual benefits in the context of cooperative relaying. This trading will be carried out by negotiating the specific number of TSs allocated, namely the value of $\beta_{l, k}$. The PUs are willing to allow the CUs to transmit using the PU-resources in exchange for the CUs assistance in transmitting the PUs' message within the number of TSs allocated, namely $\beta_{l, k}$. This would reduce the transmission duration and save power for the PU, while the $\mathrm{CU}$ may be granted a time-slot duration of $\left(1-\beta_{l, k}\right)$ for transmitting its own information. As an example, a $\beta_{l, k}$ offer is given by $\mathrm{Pt}_{l}$ to the top $\mathrm{Ct}_{k}$ in its preference list $\mathrm{PULIST}_{l}$, which grants for the $\mathrm{Ct}_{k}$ a TS of $\left(1-\beta_{l, k}\right) T$ duration for the CU's own transmission. When this $\mathrm{Ct}_{k}$ received an offer, it has two options, namely either to reject the offer, if the $\mathrm{Pt}_{l}$ is not in $\mathrm{Ct}_{k}$ 's CULIST $k$ or to accept, provided that this $\mathrm{Pt}_{l}$ is in 
the CULIST ${ }_{k}$, which leads to the matching of $\left(\mathrm{Pt}_{l}, \mathrm{Pr}_{l}\right)$ and $\left(\mathrm{Ct}_{k}, \mathrm{Cr}_{k}\right)$. If this intended $\mathrm{Ct}_{k}$ has already been matched to $\mathrm{Pt}_{c u r}$, and this $\mathrm{Pt}_{c u r}$ failed to provide a higher rate for $\mathrm{Ct}_{k}$, i.e. if we have $R_{l, \text { cur }}^{C U}\left(\beta_{l, \text { cur }}\right)<R_{l, k}^{C U}\left(\beta_{l, k}\right)$ in terms of Eq. (9), then the $\mathrm{Ct}_{k}$ will discard its current matching in favour of the new matching. Moreover, the rejected $\mathrm{Pt}_{c u r}$ will update the number of TSs allocated by setting it to $\beta_{l, c u r}^{*}=\beta_{l, c u r}-\tau$, and then it will reconstruct its preference list based on $\beta_{l, c u r}^{*}$ and repeat the matching. This algorithm aims for finding the specific number of TSs to be allocated, which can be accepted both by the $\left(\mathrm{Pt}_{l}, \mathrm{Pr}_{l}\right)$ as well as by the $\left(\mathrm{Ct}_{k}, \mathrm{Cr}_{k}\right)$ pairs, and the algorithm will be terminated when each PU pair has found its appropriate matched pair, provided that their rate requirements can be satisfied. More specifically, the CDA constitutes a non-cooperative scheme, where none of the PUs cooperates. Instead, they compete with each other, with the selfish objective of maximizing their own rate. Let us denote the average rate of $\mathrm{PU}_{l}$ in $\mathrm{CDA}$ as:

$$
r_{l}^{S}=E\left[R_{l}^{S}\right],
$$

where $E[$.$] is the expected value of [.], the superscript S$ indicates the selfish nature of the CDA and $R_{l}^{S}$ is the instantaneous rate of $\mathrm{PU}_{l}$ during a particular transmission.

3) The Proposed Pragmatic Distributed Algorithm (PDA): As mentioned in the CDA of Section III-B2, the selected Ct will reject its current matching in favour of a new matching, whenever the new matching pair is capable of providing a higher rate for the $\mathrm{Ct}$. We found that there is a drawback associated with this procedure. Assuming that there are two PUs in the system, namely $\mathrm{Pt}_{1}$ and $\mathrm{Pt}_{2}$ and that $\mathrm{Ct}_{k}$ is capable of satisfying the minimum $\mathrm{PU}$ rate requirements of both $\mathrm{Pt}_{1}$ as well as $\mathrm{Pt}_{2}$, then both $\mathrm{Pt}_{1}$ and $\mathrm{Pt}_{2}$ would list $\mathrm{Ct}_{k}$ in their preference lists of PULIST 1 and PULIST ${ }_{2}$. Then $\mathrm{Pt}_{1}$ and $\mathrm{Pt}_{2}$ will both make offers to this $\mathrm{Ct}_{k}$. Following this, there is a competition between $\mathrm{Pt}_{1}$ and $\mathrm{Pt}_{2}$. Assuming that $\mathrm{Pt}_{1}$ won and hence $\mathrm{Pt}_{2}$ was discarded. Then $\mathrm{Pt}_{2}$ will update the number of allocated TS in order to offer a higher rate for the $\mathrm{CU}$ at the cost of a lower rate for $\mathrm{Pt}_{2}$ according to the updated TS, $\beta_{l, \text { cur }}^{*}=\beta_{l, \text { cur }}-\tau$.

For the sake of ameliorating this particular situation in order to achieve higher total and individual profits for the matched PUs, we have proposed the PDA that aims for maximizing the utility of the matched PU. More specifically, our PDA is a cooperative scheme, where all PUs forms a grand coalition [23]. A game unit is constituted by $L_{P U}$ rounds and each round has $L_{P U}$ transmissions, where the PUs take turns to select the best available $\mathrm{CU}$ according to a round-robin type priority access list. The priority access list of the $i$ th round is given by:

$$
A L I S T_{i}=\left\{P t_{i}, P t_{i \oplus 1}, \ldots, P t_{l}, \ldots, P t_{i \oplus\left(L_{P U}-1\right)}\right\}
$$

where $i=\left\{1,2, \ldots, L_{P U}\right\}$ and the subscript of $P t_{l}$ for the $j$ th transmission $\left(j=\left\{1,2, \ldots, L_{P U}\right\}\right)$ in the $i$ th round is based on the modulo- $L_{P U}$ summation:

$$
l=i \oplus(j-1)=(i+(j-1)) \bmod L_{P U} .
$$

Hence, we have $A L I S T_{1}=\left\{P t_{1}, P t_{2}, P t_{3} \ldots, P t_{L_{P U}}\right\}$ and $A L I S T_{2}=\left\{P t_{L_{P U}}, P t_{1}, P t_{2}, \ldots, P t_{L_{P U-1}}\right\}$. The first Pt in the $A L I S T_{i}$ has the first priority to select its best CU. Then the second $\mathrm{Pt}$ in the list selects the best available $\mathrm{CU}$ from the remaining set of CUs, while the third Pt in the list selects its best available $\mathrm{CU}$ afterwards and the same procedure is invoked for the rest of the Pts in the list. During the next round, the first $\mathrm{Pt}$ in $A L I S T_{i}$ will become the second $\mathrm{Pt}$ in $A L I S T_{i+1}$, while the last $\mathrm{Pt}$ in $A L I S T_{i}$ is now the first Pt in $A L I S T_{i+1}$ according to the round-robin scheduling. Hence, after $L_{P U}$ rounds each $\mathrm{PU}$ is guaranteed to have access to $\min \left\{L_{P U}, L_{C U}\right\}$ CUs amongst the top CUs in its PULIST $^{5}$, but is has no access to any CUs for the remaining $\left(L_{P U}-L_{C U}\right)$ transmissions. In this way, the PUs give up any futile competition and cooperatively take turns, one at a time, to access the available CUs, which is expected to yield the most benefits for themselves. If none of the CUs in the current list may be satisfied, then only this specific Pt will update its TS allocation and then produces a new preference list. The proposed PDA has a low complexity because it does not require any exchange of information amongst the PUs, such as their rates. The first access list $A L I S T_{1}$ can be distributed at the beginning of the game by the base-station or by a PU acting as a cluster-head and the remaining lists can be computed locally by each PU. Then the PDA can be repeated automatically as many times as needed. Hence, each PU is guaranteed to have access to its top $\mathrm{CU}$ at least $1 / L_{P U}$ times $^{6}$ on average. Note that the maximum TS allocation representing the maximum transmission period for the PU can be derived from Eq. (15) as:

$$
\beta_{l, k}^{\max }=1-\frac{R_{k, r e q}^{C U}}{\log _{2}\left[1+\gamma_{C U}\left|h_{C t_{k}, C r_{k}}^{(l)}\right|^{2}\right]},
$$

while the minimum TS allocation can be computed from Eq. (4) and Eq. (5) as:

$$
\beta_{l, k}^{\min }=\frac{R_{l, r e q}^{P U}}{C_{P U_{l, k}}} .
$$

The specific details of the algorithm can be summarized as follows:

1) Initialization:

a) Set up the first priority list $A L I S T_{1}=$ $\left\{P t_{1}, P t_{2}, \ldots, P t_{L_{P U}}\right\}$ and broadcast it to all PUs.

b) Each Pt computes the remaining priority lists $A L I S T_{i}$ for $i=\left\{2,3, \ldots, L_{P U}\right\}$, based on the round-robin method given in Eq. (18).

c) Compute $\beta_{l, k}^{\min }$ and $\beta_{l, k}^{\max }$.

d) Set $i=1$ for the first round.

2) Do the matching for the $i$ th round:

a) Set the initial TS allocations to $\beta_{\text {init }}$, and set the step size of TS increment to $\tau$.

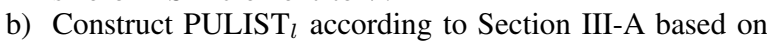
$\beta_{\text {init }}$, where $l=\left\{1, \ldots, L_{P U}\right\}$.

c) Construct $\mathrm{CU}_{\text {NOTMATCH }}=\left\{C t_{1}, \ldots, C t_{L_{C U}}\right\}_{l=1}^{L_{C U}}$ to list all participating Cts.

d) Set $j=1$ for the first transmission.

e) Do the matching for the $j$ th transmission:

i) Find the corresponding $\mathrm{Pt}_{l}$ for transmission, where $l=(i+(j-1)) \bmod L_{P U}$.

ii) $\mathrm{Pt}_{l}$ selects the best available $\mathrm{Ct}_{k}$ from $\mathrm{CU}_{N O T M A T C H}$ based on PULIST ${ }_{l}$ :

\footnotetext{
${ }^{5}$ Provided that the rate requirements in Eq. (13) and Eq. (15) are satisfied. ${ }^{6}$ The PU would have access to its top CU in all $L_{P U}$ rounds, if this CU is not sought after by other PUs.
} 
- $\mathrm{Pt}_{l}$ offers $\beta_{l, k}$ to $\mathrm{Ct}_{k}$.

- If $\beta_{l, k}^{\min } \leq \beta_{l, k} \leq \beta_{l, k}^{\max }$, then $\left(\mathrm{Pt}_{l}, \operatorname{Pr}_{l}\right)$ and $\left(\mathrm{Ct}_{k}, \mathrm{Cr}_{k}\right)$ are matched. Remove $\mathrm{Ct}_{k}$ from $\mathrm{CU}_{N O T M A T C H}$. If $\mathrm{CU}_{\text {NOTMATCH }} \in \emptyset$ goto Step 3, else go to Step 2f.

- Otherwise, reduce the TS allocation to $\beta_{l, k}=$ $\beta_{l, k}-\tau$ and update PULIST $_{l}$.

- If PULIST $\mathrm{P}_{l}$ is empty then $\mathrm{Pt}_{l}$ is left unmatched and proceed to Step $2 \mathrm{f}$.

- Otherwise, find another match at Step 2(e)ii.

f) Set $j=j+1$ and go to Step 2e for the next transmission, until $j=L_{P U}$.

3) Set $i=i+1$ and go to Step 2 for the next round, until $i=L_{P U}$.

4) Terminate the game or repeat the game from Step 1 until no more transmission is needed.

The rate of $\mathrm{PU}_{l}$ averaged over $L_{P U}$ transmissions in the $i$ th round can be computed as:

$$
R_{l}^{C}=\frac{1}{L_{P U}} \sum_{i=1}^{L} R_{l, \kappa(i)}^{P U}\left(\beta_{l, \kappa(i)}\right),
$$

where the superscript $C$ signifies the cooperative nature in PDA, $L=\min \left\{L_{P U}, L_{C U}\right\}$ and $\kappa(i)$ is the index of the best available $\mathrm{Ct}$ which satisfies the rate conditions of Eq. (13) and Eq. (15) during the $i$ th round, while $R_{l, \kappa(i)}^{P U}\left(\beta_{l, \kappa(i)}\right)=0$ if $\kappa(i) \in \emptyset$. Hence, the average rate of $\mathrm{PU}_{l}$ after many repetitions is given by:

$$
r_{l}^{C}=E\left[R_{l}^{C}\right] .
$$

The proposed PDA does not require any exchange of the PU's rate information and we assume a practical time-varying wireless channel, which may change for each transmission round and the users are also allowed to move. According to the law of large numbers, once the PDA has been repeated a sufficiently high number of times, all PUs will achieve the same average PU rate due to having random channel conditions ${ }^{7}$. More explicitly, Fig. 3 shows that when the PDA is repeated $N \geq 100$ times, the individual rates of $\mathrm{PU}_{1}$ and $\mathrm{PU}_{8}$ in the cooperative $\mathrm{CR}$ scheme would converge to the same value. This was verified for a range of scenarios having different numbers of CUs, while the number of PUs is fixed to $L_{P U}=8$. Similar trends were also observed for the other 6 PUs. Hence, the PDA is fair to all PUs, because their individual rates converged to the same value of $r_{l}^{C}$ after a sufficient number of repetitions.

4) Random Algorithm (RA): For the RA, each Pt will make an offer $\beta$ to a $\mathrm{Ct}$, which is randomly selected from its preference list. The selected $\mathrm{Ct}$ will choose that specific matching pair, which provides an increased $\mathrm{CU}$ sum rate, whilst discarding the one having a lower sum rate. More specifically, we followed the approach adopted in [12], where this $\beta$ value is fixed for all PU and SU pairs and it was chosen experimentally for the sake of maximizing the average PU sum-rate. This RA is used for benchmarking both the CDA and the proposed PDA. Note that the CA and the RA represent the two extremes in terms of their overhead and the complexity imposed.

\footnotetext{
${ }^{7}$ User mobility is considered in the channel.
}

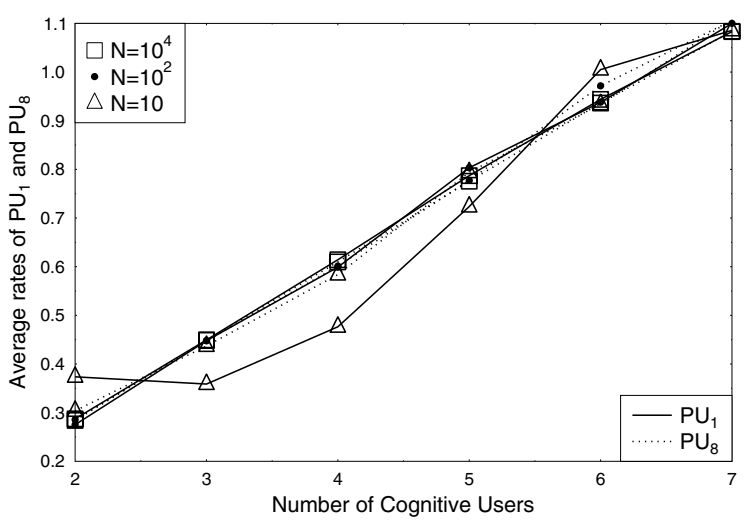

Fig. 3. Performance of $P U_{1}$ and $P U_{8}$ in the CCMC aided AAF based cooperative CR scheme when the PDA is invoked. The transmit SNRs of PU and $\mathrm{CU}$ are $\gamma_{P U}=10 \mathrm{~dB}$ and $\gamma_{C U}=25 \mathrm{~dB}$, respectively. The number of PUs is $L_{P U}=8$ and the Rayleigh fading channel condition changes for each transmission round. The number of repetitions considered in the game are $N=\left\{10,10^{2}, 10^{4}\right\}$.

\section{Repeated Game}

The CDA was shown in [12] to create a stable matching, which exhibits a competitive equilibrium, when all the PUs are non-cooperative. If we consider a 'single-shot' game, where each PU only cares about its current payoff, then no individual PU would have the incentive to deviate from the CDA strategy. Hence, the CDA may be deemed to be a strategy that arrives at an equilibrium for the one-shot non-cooperative game, having an expected one-shot payoff of $R_{l}^{S}$ given in Eq. (17). However, spectrum sharing between PUs and CUs may last for a long period of time, which may be viewed as a game repeated for many rounds, in which the PUs can cooperate based on their individual reputation and their mutual trust. More specifically, the proposed PDA is capable of guaranteeing a higher individual average PU rate compared to that of the CDA due to the avoidance of competition among the PUs. As shown in Section VI, the PDA outperforms the CDA, especially when the number of CUs is lower than that of the PUs. However, a PU using the PDA may be tempted to abandon cooperation for the sake of gaining a higher instantaneous rate. Hence, we considered a penalty/punishment based repeated game [19][21], where the PUs (players) have incentives to cooperate for the sake of achieving a higher expected payoff (average PU rate), while any non-cooperative behaviour can be avoided by appropriate punishment over a carefully selected limited period. Although the PDA may not converge to a stable equilibrium in a single-shot game, it does converge to an equilibrium in the repeated game enforced by the threat of punishment. If any of the PUs opts out of cooperation in the PDA, all PUs would revert to the non-cooperative CDA for a period of sufficiently long duration. This punishment would discourage opting out and would help to maintain cooperation.

More explicitly, the payoff of $\mathrm{PU}_{l}$ in a repeated game is defined as the sum of payoffs of $\mathrm{PU}_{l}$ discounted over time according to [19]:

$$
U_{l}=(1-\delta) \sum_{i=1}^{\infty} \delta^{i} R_{l}^{C}[i],
$$

where $\delta(0<\delta<1)$ is the discount factor and $R_{l}^{C}[i]$ is the average rate of $\mathrm{PU}_{l}$ defined in Eq. (22) for the $i$ th round. 
When we have $\delta \rightarrow 1$, the PU is more patient and hence any future reward is weighed identically to the current payoff. Hence, the PU will constrain its current behaviour in the interest of maintaining a good reputation. Let us denote the discounted payoff for cooperation in the PDA as $U_{l}^{C}$ and that for opting out of cooperation (deviation) in the CDA as $U_{l}^{D}$. Then the following proposition ${ }^{8}$ suggests that both $U_{l}^{C}$ and $U_{l}^{D}$ would converge to their means. Hence, it is better to maintain cooperation for each PU's benefit, as long as, we have $r_{l}^{C}>r_{l}^{S}$.

Proposition 1: As we have $\delta \rightarrow 1$, the instantaneous payoff $U_{l}^{C}$ would converge to the expected payoff $r_{l}^{C}$ while the current payoff $U_{l}^{D}$ would converge to the averaged payoff $r_{l}^{S}$.

On the other hand, imposing an infinite-duration punishment is not efficient for all PUs, because all of them would be punished and would only result in a reduced PU rate of $r_{l}^{S}$. Explicitly, a limited-duration punishment [19] is a more efficient way of preventing non-cooperation, as long as the punishment is long enough to negate the one-time non-cooperation gain. If any PU deviates from cooperation in the PDA, then all PUs would revert to the non-cooperative CDA for the next $T_{p}$ instances. Next, we show in the following proposition ${ }^{9}$ that the limited-duration punishment based cooperation in PDA also has a perfect subgame equilibrium, which ensures optimality for subgames starting from any round of the entire repeated game.

Proposition 2: Provided that $r_{l}^{C}>r_{l}^{S}$ for all $l, l \in$ $\left\{1,2, \ldots, L_{P U}\right\}$, we have $\tilde{\delta}<1$, so that for a sufficiently large discount factor $\delta>\tilde{\delta}$, the game has a perfect subgame equilibrium with a discounted utility of $r_{l}^{C}$, provided that all players are governed by the limited-punishment strategy.

The punishment period duration $T_{p}$ can be determined by analysing the conditions under which a PU would abandon cooperation at instant $T^{*}$. Consider the extreme case, where the payoff of $\mathrm{PU}_{l}$ at instant $T^{*}$ would be $R_{l, r e q}^{P U}=C_{P U}^{*}$ of Eq. (8), when no $\mathrm{CU}$ was matched to $\mathrm{PU}_{l}$ under the PDA, while the deviation gain ${ }^{10}$ would be $\tilde{R}_{l}^{D}$. The expected discounted payoffs for $\mathrm{PU}_{l}$ derived both with and without noncooperation are bounded by:

$$
\begin{aligned}
u_{l}^{D}=E\left[U_{l}^{D}\right] \leq & (1-\delta) \cdot\left(\sum_{i=1}^{T^{*}-1} \delta^{i} r_{l}^{C}+\delta^{T^{*}} \tilde{R}_{l}^{D}+\right. \\
& \left.\sum_{i=T^{*}+1}^{T^{*}+T_{p}} \delta^{i} r_{l}^{S}+\sum_{i=T^{*}+T_{p}+1}^{\infty} \delta^{i} r_{l}^{C}\right)
\end{aligned}
$$

and

$$
\begin{aligned}
u_{l}^{C}=E\left[U_{l}^{C}\right] \geq & (1-\delta) \cdot\left(\sum_{i=1}^{T^{*}-1} \delta^{i} r_{l}^{C}+\delta^{T^{*}} R_{l, r e q}^{P U}+\right. \\
& \left.\sum_{i=T^{*}+1}^{T^{*}+T_{p}} \delta^{i} r_{l}^{C}+\sum_{i=T^{*}+T_{p}+1}^{\infty} \delta^{i} r_{l}^{C}\right),
\end{aligned}
$$

\footnotetext{
${ }^{8}$ The proof is given in Appendix A.

${ }^{9}$ The proof is given in Appendix B.

${ }^{10} \mathrm{We}$ assume a hypothetical scenario, where $\mathrm{PU}_{l}$ is capable of predicting its payoff $\left(\mathrm{PU}_{l}\right.$ rate) under $\mathrm{CDA}$, although the $\mathrm{PU}$ rate is only available at the end of the PU competition in the CDA.
}

respectively. A selfish PU would prefer a strategy that can provide a higher payoff. It can be shown based on Eq. (25) and Eq. (26) that the requirement for $u_{l}^{C}>u_{l}^{D}$ for all $l$ would lead to the following condition:

$$
\sum_{i=1}^{T_{p}} \delta^{i}>\max _{l} \frac{\tilde{R}_{l}^{D}-R_{l, r e q}^{P U}}{r_{l}^{C}-r_{l}^{S}}
$$

where we have $\sum_{i=1}^{T_{p}} \delta^{i} \rightarrow T$, when $\delta \rightarrow 1$. Hence, the punishment period is bounded by:

$$
T_{p}>\max _{l} \frac{\tilde{R}_{l}^{D}-R_{l, r e q}^{P U}}{r_{l}^{C}-r_{l}^{S}}
$$

In other words, as long as $T_{p}$ satisfies Eq. (28), $\mathrm{PU}_{l}$ would not deviate from the cooperative strategy, since the one-time payoff under the non-cooperative strategy has been negated by punishment, so that we have $u_{l}^{C}>u_{l}^{D}$.

Hence, the repeated game based on the PDA is capable of providing higher individual PU rates as well as a higher PU sum-rate on average. Hence it is an attractive and stable game, even when no information is available about PU rates. We now further develop and analyse this game for employment in systems relying on realistic coding and modulation schemes in the next section.

\section{Coding And Modulation Design}

In our proposed system, we will make use of this powerand bandwidth-efficient TTCM scheme. Employing TTCM has the advantage that the system's effective throughput can be increased upon increasing the code rate, when the channelquality improves. Additionally, the Bit error ratio (BER) performance of the system may be improved when TTCM is used [31]-[33]. The TTCM encoder comprises two identical parallel-concatenated TCM encoders [26] linked by a symbol interleaver. The first TCM encoder directly processes the original input bit sequence, while the second TCM encoder manipulates the interleaved version of the input bit sequence. Then the bit-to-symbol mapper maps the input bits to complexvalued symbols using the Set Partition (SP)-based labelling method [32]. The structure of the TTCM decoder is similar to that of binary turbo codes, but each decoder alternately processes its corresponding encoder's channel-impaired output symbol, and then the other encoder's channel-impaired output symbol [32, p.764]. More details on the TTCM principles may be found in [32]. We have employed a Adaptive TTCM (ATTCM) scheme for protecting the SR and the RD links, where the effective throughput (or information Bit-perSymbol (iBPS) ) range is given by $\mathrm{iBPS}=\{0,1,2,3,5\}$ bps when no transmission, QPSK, 8PSK, 16QAM and 64QAM are considered, respectively. Moreover, the TTCM mode switching thresholds $\Upsilon=\left[\gamma_{0}, \gamma_{1}, \gamma_{2}, \gamma_{3}\right]$ were determined based on the BER performance curves of each of the four TTCM schemes in a Rayleigh fading channel, which is shown in Fig. 4. Specifically, the ATTCM mode switching operation and the throughput of the modes are specified by the following 
algorithm:

$$
\text { MODE }= \begin{cases}\gamma_{3} \leq \gamma_{R}, & \text { TTCM-64QAM, BPS=5 bps; } \\ \gamma_{2} \leq \gamma_{R}<\gamma_{3}, & \text { TTCM-16QAM, BPS=3 bps; } \\ \gamma_{1} \leq \gamma_{R}<\gamma_{2}, & \text { TTCM-8PSK, BPS=2 bps; } \\ \gamma_{0} \leq \gamma_{R}<\gamma_{1}, & \text { TTCM-4PSK, BPS=1 bps; } \\ \gamma_{R}<\gamma_{0}, & \text { No transmission, BPS=0 bps. }\end{cases}
$$

In this section we investigate the practical design of the cooperative CR scheme advocated using ATTCM based on the DAF protocol. As shown in Fig. 1, all Cts/RN operate in the half-duplex DAF mode and it is assumed that each $\mathrm{Ct} / \mathrm{RN}$ only knows its own channel, but that the Pr estimated all channels with the aid of training. The signal received via the direct (Pt-Pr) link is also detected. We use the notation $\gamma_{a b}$ to refer to the instantaneous receive SNR of the link between node $a$ and node $b$. The receive SNR at node $b$ is given by:

$$
\gamma_{R}=\frac{\varrho\left|h_{a b}\right|^{2}}{N_{0}},
$$

where $h_{a b}$ represents the quasi-static Rayleigh fading channel between nodes $a$ and $b$. The channel gains are independent of each other. The quasi-static Rayleigh fading channels between the Pts and the Cts/RN are denoted as $\left[h_{P t_{1}, C t_{1}}, \ldots, h_{P t_{l}, C t_{k}}\right]_{L_{P U} \times L_{C U}}$, while those between the Cts/RNs and the Prs are represented by $\left[h_{C t_{1}, P r_{1}}, \ldots, h_{C t_{l}, P r_{k}}\right]_{L_{C U} \times L_{P U}}$. As seen from Fig. 1, we have $(l \times k)$ links spanning from the Pts to the $\mathrm{Ct} / \mathrm{RNs}$ supported by $K \mathrm{Cts} / \mathrm{RN}$ and also $(k \times l)$ links spanning from the Cts/RN to the Prs.

Each of the communication links will be assisted by the ATTCM scheme. We chose the switching thresholds to ensure that the BER at the RN is lower than $10^{-5}$, which is given by $\Upsilon_{A T T C M}=[4.8,12,16,24] \mathrm{dB}$ as seen in Fig. 4. We note that Shannon's CCMC capacity is only restricted by the SNR and the bandwidth. The CCMC-based switching thresholds are represented as $\Upsilon_{C C M C}=[1.75,6,11,14] \mathrm{dB}$, while the switching thresholds of the corresponding modulation dependent DCMC based scheme are given by $\Upsilon_{D C M C}=[2,8,12.5$, 20] dB, which are also explicitly shown in Fig. 4. The reason why we have chosen the BER at the RN to be lower than $10^{-5}$ is because the error floor emerging at $\mathrm{BER}<10^{-5}$ can be removed by using a long outer code, such as a Reed Solomon (RS) code, albeit no RS code was used here.

For quasi-static fading channels, the achievable rates over different links become random and vary as the channel changes. We have considered two different methods of evaluating the total system rate $R_{\text {total }}$ between the communication link Pt-Ct and Ct-Pr, namely both the lower-bound and upperbound of the system's total rate $R_{\text {total }}$. The lower bound assumes that the DAF based RN/Ct forwards the received signal to $\mathrm{Pr}$ directly and the corresponding rate is the minimum of the rates in the Pt-Ct and Ct-Pr links [34], [35]:

$$
R_{l, k}^{\text {lower }}=\frac{1}{t} \min \left\{R_{P t_{l}-C t_{k}}, R_{C t_{k}-P r_{l}}\right\},
$$

where $t$ is the number of TSs in the cooperative relay transmission. We consider the classical $t=2$ TSs based cooperative relaying channel. We ensured that the specific hop associated with the lower sum rate would convey its message

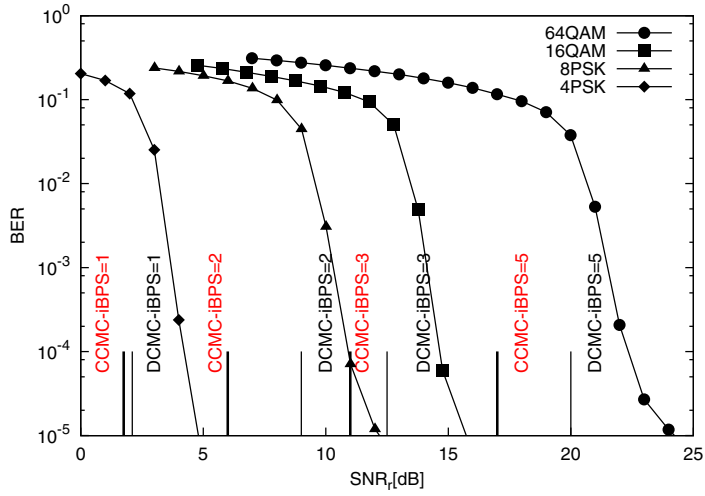

Fig. 4. The BER versus $\mathrm{SNR}_{r}$ performance of TTCM using a frame length of 120,000 symbols, when communicating over Rayleigh channels. Four TTCM iterations were invoked.

TABLE II

THE PARAMETERS OF OUR PROPOSED ATTCM AIDED COOPERATIVE CR SCHEME.

\begin{tabular}{|l|r|}
\hline Modulation & 4-PSK, 8-PSK, 16-QAM, 64-QAM \\
\hline Coding & TTCM \\
\hline Number of frames & $10^{5}$ \\
\hline Channel & Rayleigh fading channel \\
\hline Total number of CU & $L_{C U}=10$ \\
\hline Total number of PU & $L_{P U}=8$ \\
\hline Initialization of $\beta$ for CDA & $\beta_{\text {init }}=0.99$ \\
\hline Initialization of $\beta$ for PDA & $\beta_{\text {init }}=\beta_{\max }$ \\
\hline Step size of $\beta$ & $\tau=0.05$ \\
\hline Pathloss exponent & $\alpha=4$ \\
\hline Requirement of CU & $R_{r e q}^{C U}=2.0$ \\
\hline Requirement of PU & $R_{r e q}^{P U}=C_{P U}^{*}$ \\
\hline Pathloss & $\varrho=\frac{1}{d^{\alpha}}$ \\
\hline
\end{tabular}

successfully, while the other hop having the higher sum rate would give up some of its capacity. Moreover, we have also considered the upper-bound of the total achievable rate based on the average of the achievable sum-rates of both the Pt-Ct and Ct-Pr links as [36]:

$$
R_{l, k}^{u p p e r}=\frac{1}{t}\left(R_{P t_{l}-C t_{k}}+R_{C t_{k}-P r_{l}}\right) .
$$

More specifically, the upper bound assumes that the DAF based RN/Ct always has enough data for transmission to the Pr and that its memory is always sufficient for storing the data arriving from the Pt.

\section{Vi. Performance Results}

\section{A. Performance of the matching algorithm aided cooperative CR network}

Fig. 5(a) shows the average total sum-rate of the matched $(\mathrm{Pt}, \mathrm{Pr})$ pairs versus the total number of CUs, $L_{C U}=$ $\{2,3, \ldots, 10\}$, for the proposed cooperative CR scheme, when we have $L_{P U}=8$. In our evaluation of Eq. (5), Eq. (8) and Eq. (9), we assumed that the transmit SNRs of all CUs are equal, yielding $\gamma_{C U_{1}}=\cdots=\gamma_{C U_{l}}=\gamma_{C U}$. We also assumed that the SNR of all PUs are $\gamma_{P U_{1}}=\gamma_{P U_{2}}=10 \mathrm{~dB}$. The remaining simulation parameters are shown in Table II. We investigate our scheme in conjunction with two different transmit SNRs of the CU, namely for $\gamma_{C U}=15 \mathrm{~dB}$ and $\gamma_{C U}=25 \mathrm{~dB}$. When $\gamma_{C U}$ is increased, the average total sum-rate of the matched (Pt, Pr) pairs is also increased. 


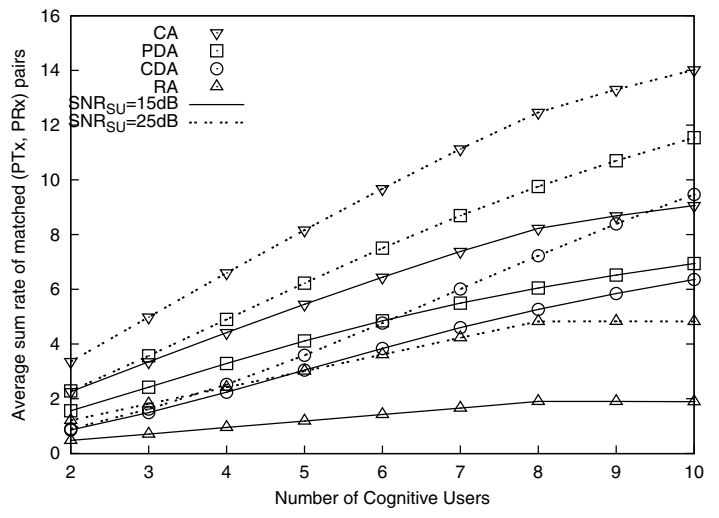

(a) $R_{\text {match }}^{P U}$ versus $L_{C U}$

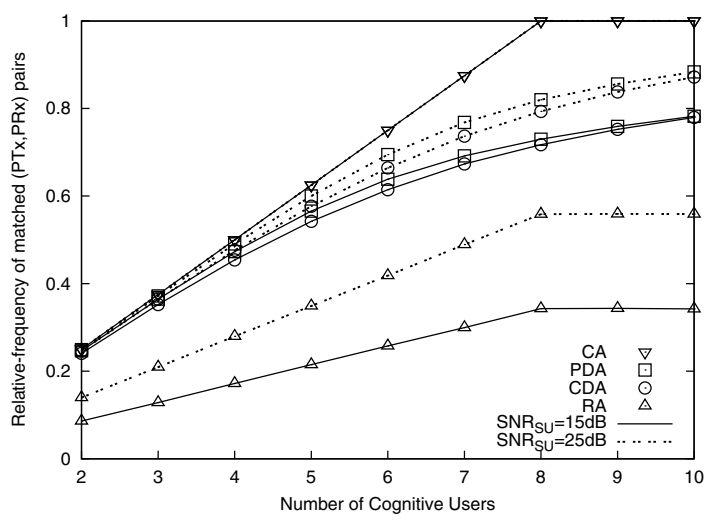

(b) $P_{\text {match }}$ versus the $L_{C U}$

Fig. 5. Performance of the CCMC aided AAF based cooperative CR benchmark [12] scheme communicating over block-fading Rayleigh fading channel. A BER below $10^{-5}$ is maintained. The "CA", "PDA", "CDA" and "RA" techniques were detailed in Section III-B. The transmit SNR of the $\mathrm{CU}, \gamma_{C U}$, is represented by $S N R_{C U}$.

We have considered the $\mathrm{CDA}$, the PDA, the $\mathrm{CA}$ and the RA of Section III-B4 in Fig. 5(a). The CA achieves the highest average total sum-rate among these four algorithms, while the RA achieves the lowest sum rate in Fig. 5(a). It is observed in Fig. 5(a) that our PDA achieves a higher sumrate than that of the CDA at the same $L_{C U}$ and $\gamma_{C U}$. The PDA consistently attains a higher rate than the CDA for all scenarios. Furthermore, when we have $\gamma_{C U}=25 \mathrm{~dB}$ and $L_{C U} \leq 3$, the CDA scheme performs slightly worse than the RA scheme, due to the competition loss encountered.

Fig. 5(b) portrays the expected relative frequency of successfully matched PUs versus the number of CUs, which is evaluated as:

$$
P_{\text {match }}=\frac{1}{L_{P U}} E\left[\sum_{l}^{L_{P U}} \sum_{k}^{L_{C U}} m_{l, k}\right] .
$$

It is observed in Fig. 5(b) that $P_{\text {match }}$ increases upon increasing $\gamma_{C U}$ and $L_{C U}$, because a higher sum-rate was achieved by the matched (Pt, Pr) pairs with the aid of cooperative relaying, when there are more CUs available and the channel quality improves. As shown in Fig. 5(b), the $P_{\text {match }}$ of the CA is the highest, followed by that of the PDA, CDA and RA. Hence, the rate-improvement observed in Fig. 5(a) is linked to a higher $P_{\text {match }}$, resulting from a better matching.

Fig. 6(a) and Fig. 6(b) show the average total sum-rate of

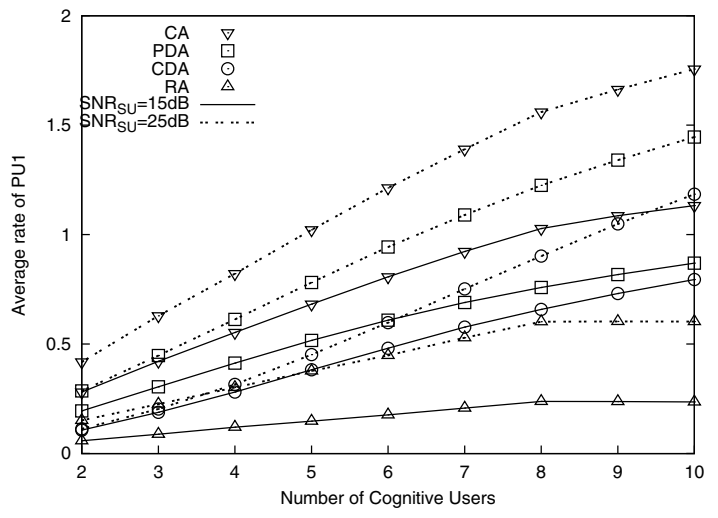

(a) Average Rate of $\mathrm{PU}_{1}$

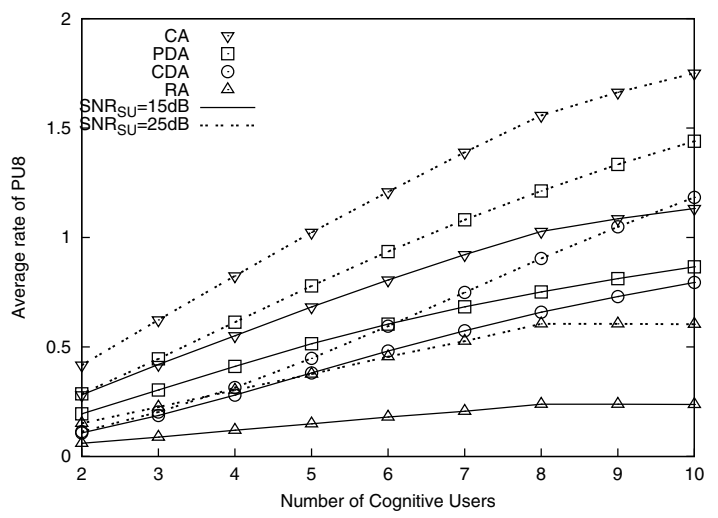

(b) Average Rate of $\mathrm{PU}_{8}$

Fig. 6. $R_{\text {match }}^{P U}$ versus $L_{C U}$ performance of the CCMC aided AAF based cooperative CR benchmark [12] scheme communicating over block-fading Rayleigh fading channel. A BER below $10^{-5}$ is maintained. The "CA", "PDA", "CDA" and "RA" techniques were detailed in Section III-B. The transmit SNR of the CU, $\gamma_{C U}$, is represented by $S N R_{C U}$.

the matched $\mathrm{PU}_{1}$ and $\mathrm{PU}_{8}$, versus the total number of CUs, $L_{C U}$, for the various cooperative CR schemes. As seen from Fig. 6(a), the average individual rate of $\mathrm{PU}_{1}$ and $\mathrm{PU}_{8}$ (and of all other PUs as well) has converged to the same curve after a longer simulation consisting of $10^{5}$ transmissions. We observe that the performance of $\mathrm{PU}_{l}$ (for $l \in\left\{1, \ldots, L_{P U}\right\}$ ) is close to that of the CA, when invoking the proposed PDA. By contrast, the rate of $\mathrm{PU}_{l}$ is lower, when employing the CDA because it has to make further sacrifices by reducing its TS $\beta_{l, k}$, when competing with other PUs for the same CU. Furthermore, the rate of $\mathrm{PU}_{l}$ operating under our PDA is lower than that of the CA, but much higher than that of the CDA. Hence, the proposed PDA outperforms the CDA in terms of both the total PU sum rate and the individual PU rate. Moreover, we also consider the corresponding average total sum-rate of all matched (PT, PR) pairs as a function of the step size $\tau$ in Fig. 7. As expected, the total sum-rate decreases upon increasing $\tau$. However, a small $\tau$ would lead to a longer matching period. Hence, we have chosen $\tau=0.05$ as a compromise.

\section{B. Performance of our ATTCM aided Cooperative CR Net- work}

In this section, we investigate the performance of the CCMC, DCMC and ATTCM aided DAF based cooperative 


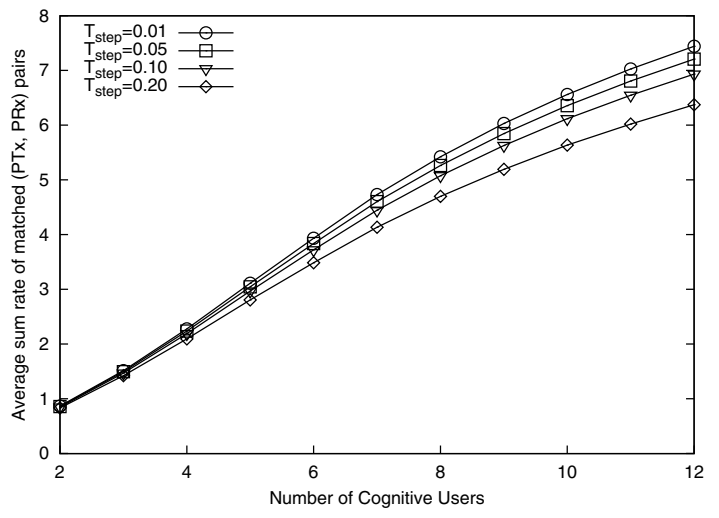

Fig. 7. Performance of the CDA aided cooperative CR scheme for the CCMC capacity. " $T_{\text {step }}$ " is the step size $\tau$ of the TS-increment. The transmit SNR of the $\mathbf{C U}$ is $\gamma_{C U}=15 \mathbf{d B}$.

CR schemes for four different matching algorithms when aiming for spectral access. The average total sum-rate of all matched $(\mathrm{Pt}, \mathrm{Pr})$ pairs versus $L_{C U}$ curves of the CCMC, DCMC and ATTCM aided cooperative CR schemes when $L_{P U}=8$, are shown in Fig. 8(a), Fig. 9(a) and Fig. 10(a), respectively. As seen from Fig. 8(a), the total sum-rate of all matched (Pt, Pr) pairs is higher, when we consider the CA of Section III-B1. We have considered both the upper-bound and lower-bound performance seen in Fig. 8(a). For the lowerbound performance, we considered the lowest transmission rate among the Pt-Ct and Ct-Pr links in order to ensure that the information of both links can be transmitted successfully. However in this case the link associated with the higher rate generously surrenders some of its unused rate for ensuring that the one with a lower rate can also transmit successfully. Additionally, the upper bound is derived, when considering the sum rate of both the Pt-Ct and Ct-Pr links, which gives a higher average total sum rate. Furthermore, as $L_{C U}$ increases, the sum-rate of all matched $(\mathrm{Pt}, \mathrm{Pr})$ pairs also increases. This is because when the total number of CUs is high, each Pt will have a high number of choices for selecting better CUs. Thus, the probability of successful matching of PUs and CUs also increases. Additionally, the final matched (Pt, Pr) pairs are the ones with the higher sum-rate. Some unmatched CUs will not be able to transmit. However, when we have $L_{C U}<L_{P U}$, the CDA-based scheme suffers from severe competition loss. More specifically, as seen for the ATTCM aided scheme in Fig. 10(a), the upper bound PU rate of the CDA scheme is even lower than that of the RA scheme, when $L_{C U} \leq 7$.

As expected, the system-rate of the matched PUs relying on the CCMC aided cooperative CR scheme is better in comparison to that of the DCMC and of the ATTCM aided schemes, as revealed in Fig. 8(a), Fig. 9(a) and Fig. 10(a). It is observed from these figures that the performance of our PDA is close to that of the CA. However, the achievable rate of matched PUs was (17.69 bps $-7.48 \mathrm{bps})=10.21 \mathrm{bps}$ higher than that of the CDA for our PDA as shown in Fig. 10(a), when we have $L_{C U}=6$. The percentage $P_{\text {match }}$ of matched PUs versus the number of CUs, $L_{C U}$, is investigated in Fig. 8(b), Fig. 9(b) and Fig. 10(b). The idealistic CCMC aided cooperative $\mathrm{CR}$ scheme achieves the highest matching percentage. Additionally, the percentage of matched users found by the CA is seen to be significantly higher than that of

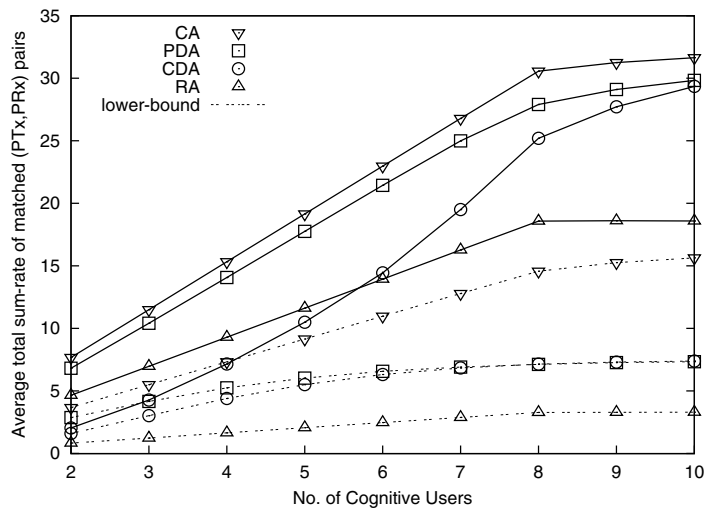

(a) $R_{\text {match }}^{P U}$ versus $L_{C U}$

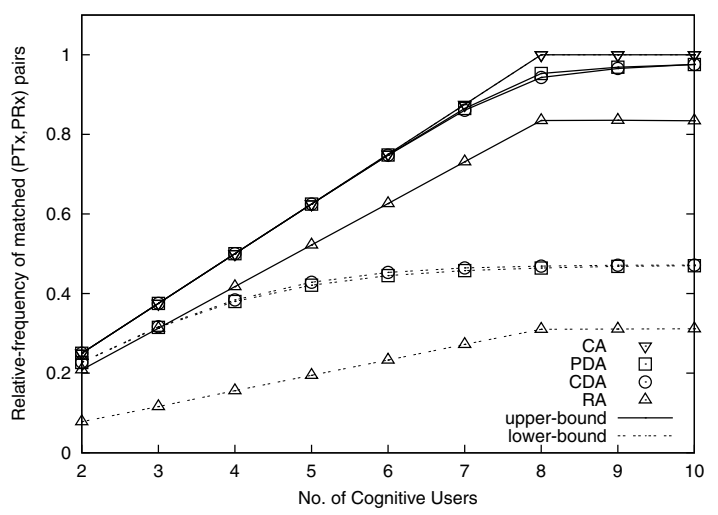

(b) $P_{\text {match }}$ versus the $L_{C U}$

Fig. 8. Performance of the CCMC aided DAF based cooperative CR scheme communicating over block-fading Rayleigh fading channel. The "CA", "PDA", "CDA" and "RA" techniques were discussed in Section III-B. The "upper-bound" and "lower-bound" refer to the upper-bound and lowerbound of the total system's throughput for transmission over the cooperative relay channel of Section V. A BER below $10^{-5}$ is maintained and the transmit SNR of PU $\gamma_{P U}=20 d B$ and the transmit SNR of CU $\gamma_{C U}=35 \mathbf{~ d B}$ is used.

the RA. Interestingly, the upper bound $P_{\text {match }}$ curves seen in Fig. 8(b) for all CA, PDA and CDA schemes are quite close to each others, although the PU rate of CDA is much lower, when $L_{C U}<L_{P U}$. This indicates that the CDA suffers from competition loss, despite having a similar $P_{\text {match }}$.

\section{CONCLUSIONS}

We have considered four PU/CU matching algorithms conceived for spectral access in our cooperative CR scheme. In our proposed PDA, the PUs trade with the CUs by negotiating the TS allocation, which guarantees that the rate requirements of the matched PUs and CUs are satisfied. We showed that the proposed PDA is capable of converging to an equilibrium in the repeated game, which is a benefit of imposing a carefully chosen limited punishment period. Our numerical analysis revealed that the proposed PDA achieved a better performance than the CDA benchmark scheme, especially when the number of CUs is lower than that of the PUs. Furthermore, the proposed PDA has a low complexity, because it does not require the sharing of information regarding the $\mathrm{PU}$ or $\mathrm{CU}$ rates amongst the PUs. If all information about the PU and $\mathrm{CU}$ rates are available, then the $\mathrm{CA}$ can be used under the 


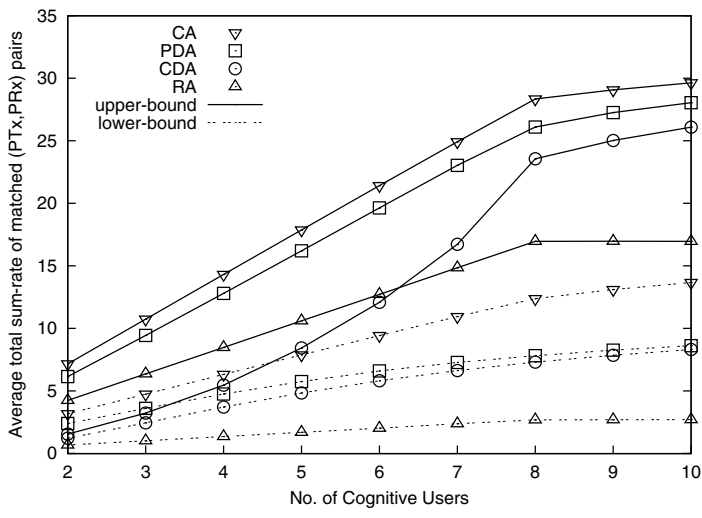

(a) $R_{\text {match }}^{P U}$ versus $L_{C U}$

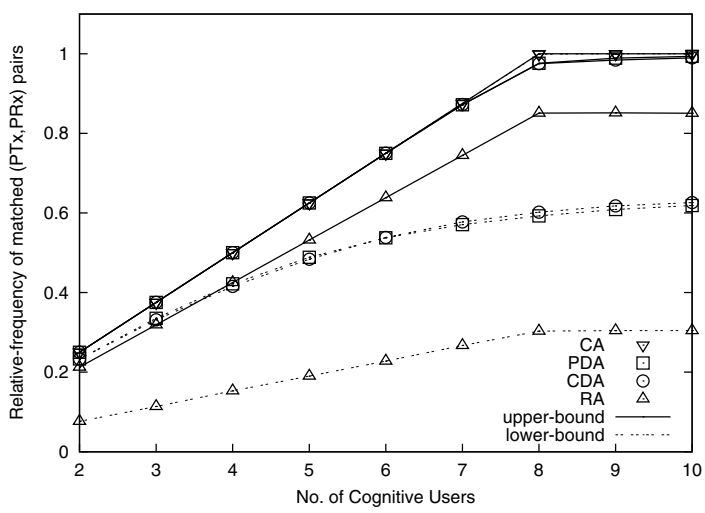

(b) $P_{\text {match }}$ versus the $L_{C U}$

Fig. 9. Performance of the DCMC aided DAF based cooperative CR scheme communicating over block-fading Rayleigh fading channel. The "CA", "PDA", "CDA" and "RA" techniques were discussed in Section III-B. The "upper-bound" and "lower-bound" refer to the upper-bound and lowerbound of the total system's throughput for transmission over the cooperative relay channel of Section V. A BER below $10^{-5}$ is maintained and the transmit SNR of PU $\gamma_{P U}=20 d B$ and the transmit SNR of CU $\gamma_{C U}=35 \mathbf{~ d B}$ is used.

repeated game concept for attaining the optimal performance with the aid of PU cooperation.

\section{APPENDIX}

\section{Appendix A: Proof of Proposition 1}

Proof: The discounted payoff in Eq. (24) can be shown to be asymptotically equivalent to the average of the one-time payoffs, when $\delta$ approaches unity as follows:

$$
\begin{aligned}
\lim _{\delta \rightarrow 1} U_{l} & =\lim _{\delta \rightarrow 1} \lim _{N \rightarrow \infty} \frac{1-\delta}{1-\delta^{N}} \sum_{i=1}^{N} \delta^{i} R_{l}^{C}[i] \\
& =\lim _{N \rightarrow \infty} \sum_{i=1}^{N}\left(\lim _{\delta \rightarrow 1} \frac{\delta^{i}-\delta^{i+1}}{1-\delta^{N}}\right) R_{l}^{C}[i] \\
& =\lim _{N \rightarrow \infty} \frac{1}{N} \sum_{i=1}^{N} R_{l}^{C}[i]=r_{l}^{C}
\end{aligned}
$$

where the last equality is derived from L'Hôpital's rule. Furthermore, if $\mathrm{PU}_{l}$ opts out of cooperation at instant $T^{*}$, then its payoff would be given by $\left\{R_{l}[i], i=0,1, \ldots, T^{*}-1\right\}$, which are i.i.d. random variables due to i.i.d. random channel variations and its mean is given by $r_{l}^{C}$ of Eq. (23). The payoff

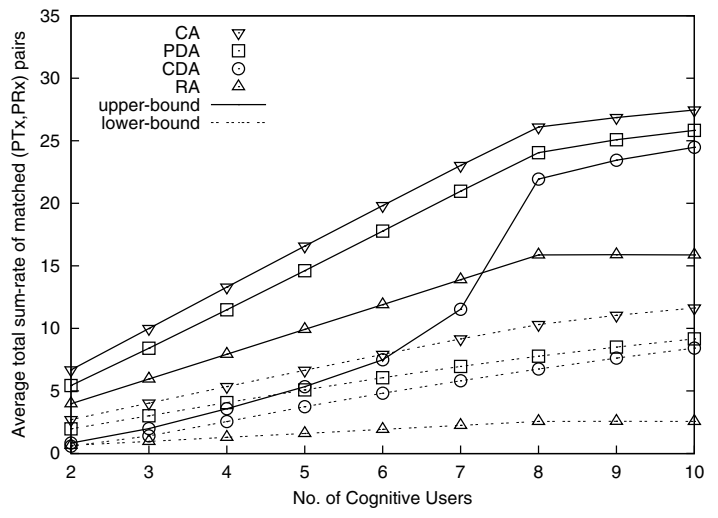

(a) $R_{\text {match }}^{P U}$ versus $L_{C U}$

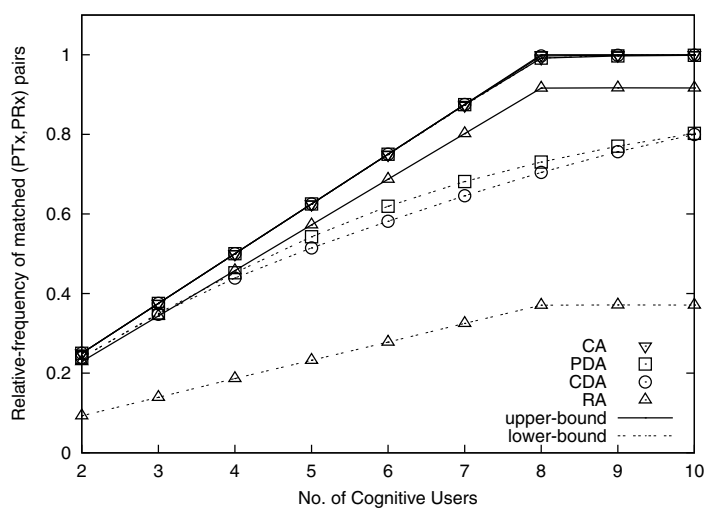

(b) $P_{\text {match }}$ versus the $L_{C U}$

Fig. 10. Performance of the ATTCM aided DAF based cooperative CR scheme communicating over block-fading Rayleigh fading channel. The "CA", "PDA", "CDA" and "RA" techniques were discussed in Section III-B. The "upper-bound" and "lower-bound" refer to the upper-bound and lowerbound of the total system's throughput for transmission over the cooperative relay channel of Section V. A BER below $10^{-5}$ is maintained and the transmit SNR of PU $\gamma_{P U}=20 d B$ and the transmit SNR of CU $\gamma_{C U}=35 \mathbf{d B}$ is used.

after the deviation is given by $\left\{R_{l}[i], i=T^{*}+1, T^{*}+2, \ldots\right\}$ which is an i.i.d. random variable with the mean $r_{l}^{S}$ of Eq. (17). The payoff after abandoning cooperation converges to its mean, $U_{l}^{D} \rightarrow r_{l}^{S}$, due to the law of large numbers. Hence, abandoning cooperation only benefits $\mathrm{PU}_{l}$ at instant $T^{*}$. Similarly, the payoff $\left\{R_{l}[i], i=0,1, \ldots\right\}$ would converge to its mean, $U_{l}^{C} \rightarrow r_{l}^{C}$ if abandoning cooperation never happens.

\section{Appendix B: Proof of Proposition 2}

Proof: Since the CDA has an equilibrium for the one-shot game, all PUs would not disagree to adopt the CDA strategy for the punishment stage. Since the CDA would result in $r_{l}^{S}$, which is lower than $r_{l}^{C}$, the threat of using the CDA strategy as the punishment after any non-cooperation would result in only a one-time gain for the 'defector', which can be readily negated by limited-duration punishment, when we have $\delta \rightarrow 1$. Hence, all PUs are motivated to adopt the cooperative strategy of the PDA throughout the game, which is also the optimal behaviour for any subgame ${ }^{11}$.

\footnotetext{
${ }^{11}$ This proof is adopted from Section 14.8 of [19].
} 
IEEE TRANSACTIONS ON COMMUNICATIONS, ACCEPTED FOR PUBLICATION

\section{REFERENCES}

[1] F. K. Jondral, "Cognitive radio: a communications engineering view," IEEE Wireless Commun., vol. 14, pp. 28-33, Aug. 2007.

[2] J. Mitola and G. Q. Maguire, "Cognitive radio: making software radios more personal," IEEE Personal Commun., vol. 6, pp. 13-18, Aug. 1999.

[3] N. Devroye, P. Mitran, and V. Tarokh, "Achievable rates in cognitive radio channels," IEEE Trans. Inf. Theory, vol. 52, pp. 1813-1827, May 2006.

[4] A. Goldsmith, S. Jafar, I. Maric, and S. Srinivasa, "Breaking spectrum gridlock with cognitive radios: an information theoretic perspective," Proc. IEEE, vol. 97, pp. 894-914, May 2009.

[5] A. Sendonaris, E. Erkip, and B. Aazhang, "User cooperation diversitypart I: system description," IEEE Trans. Commun., vol. 51, pp. 19271938, Nov. 2003.

[6] J. Laneman, D. Tse, and G. Wornell, "Cooperative diversity in wireless networks: efficient protocols and outage behavior," IEEE Trans. Inf. Theory, vol. 50, pp. 3062-3080, Dec. 2004.

[7] K. B. Letaief and W. Zhang, "Cooperative communications for cognitive radio networks," Proc. IEEE, vol. 97, pp. 878-893, May 2009.

[8] O. Simeone, J. Gambini, Y. Bar-Ness, and U. Spagnolini, "Cooperation and cognitive radio," in Proc. 2007 IEEE International Conference on Communications, pp. 6511-6515.

[9] W. Su, J. Matyjas, and S. Batalama, "Active cooperation between primary users and cognitive radio users in heterogeneous ad-hoc networks," IEEE Trans. Signal Process., vol. 60, pp. 1796-1805, Apr. 2012.

[10] Y. Han, A. Pandharipande, and S. Ting, "Cooperative decode-andforward relaying for secondary spectrum access," IEEE Trans. Wireless Commun., vol. 8, pp. 4945-4950, Oct. 2009.

[11] O. Simeone, I. Stanojev, S. Savazzi, Y. Bar-Ness, U. Spagnolini, and R. Pickholtz, "Spectrum leasing to cooperating secondary ad hoc networks," IEEE J. Sel. Areas Commun., vol. 26, pp. 203-213, Jan. 2008.

[12] S. Bayat, R. Louie, Y. Li, and B. Vucetic, "Cognitive radio relay networks with multiple primary and secondary users: distributed stable matching algorithms for spectrum access," in Proc. 2011 IEEE International Conference on Communications, pp. 1-6.

[13] S. H. Lee, M. Shamaiah, S. Vishwanath, and H. Vikalo, "A messagepassing algorithm for spectrum access in cognitive radio relay networks," in Proc. 2011 Asilomar Conference on Signals, Systems and Computers, pp. 1753-1757.

[14] M. Shamaiah, S. Lee, S. Vishwanath, and H. Vikalo, "Distributed algorithms for spectrum access in cognitive radio relay networks," IEEE J. Sel. Areas Commun., vol. 30, pp. 1947-1957, Nov. 2012.

[15] P. Lin, J. Jia, Q. Zhang, and M. Hamdi, "Dynamic spectrum sharing with multiple primary and secondary users," IEEE Trans. Veh. Technol., vol. 60, pp. 1756-1765, May 2011.

[16] D. Bertsekas, "A distributed asynchronous relaxation algorithm for the assignment problem," in Proc. 1985 IEEE Conference on Decision and Control,, vol. 24, pp. 1703-1704.

[17] C. An, L. Zhang, and W. Liu, "A spectrum allocation algorithm based on matching game," in Proc. 2009 International Conference on Wireless Communications, Networking and Mobile Computing, pp. 1-3.

[18] V. Asghari and S. Aissa, "Resource management in spectrum-sharing cognitive radio broadcast channels: adaptive time and power allocation," IEEE Trans. Commun., vol. 59, no. 5, pp. 1446-1457, 2011.

[19] M. J. Osborne, An Introduction to Game Theory. Oxford University Press, 2004.

[20] R. J. Aumann and M. Maschler, Repeated Games with Incomplete Information. The MIT Press, 1995.

[21] Y. Wu, B. Wang, K. J. R. Liu, and T. C. Clancy, "Repeated open spectrum sharing game with cheat-proof strategies," IEEE Trans. Wireless Commun., vol. 8, pp. 1922-1933, Apr. 2009.

[22] R. B. Myerson, Game Theory Analysis of Conflict. Harvard University Press, 1997.

[23] W. Saad, Z. Han, M. Debbah, A. Hjorungnes, and T. Basar, "Coalitional game theory for communication networks," IEEE Signal Process. Mag., vol. 26, no. 5, pp. 77-97, 2009.

[24] C. Wong and L. Hanzo, "Upper-bound performance of a wide-band adaptive modem," IEEE Trans. Commun., vol. 48, no. 3, pp. 367-369, 2000.

[25] S. X. Ng and L. Hanzo, "On the MIMO channel capacity of multidimensional signal sets," IEEE Trans. Veh. Technol., vol. 55, pp. 528-536, Mar. 2006.

[26] G. Ungerböck, "Channel coding with multilevel/phase signals," IEEE Trans. Inf. Theory, vol. IT-28, pp. 55-67, Jan. 1982.

[27] K. J. R. Liu, A. K. Sadek, W. Su, and A. Kwasinski, Cooperative Communications and Networking. Cambridge University Press, 2009.
[28] B. Zhao and M. C. Valenti, "Distributed turbo coded diversity for relay channel," Electron. Lett., vol. 39, pp. 786-787, May 2003.

[29] A. T. Fouad and M. M. Hira, "Joint optimization of physical layer parameters and routing in wireless mesh networks," in Proc. 2010 IFIP Mediterranean, Ad Hoc Networking Workshop (Med-Hoc-Net), pp. 1-8.

[30] S. Bayat, R. Louie, Z. Han, Y. Li, and B. Vucetic, "Distributed stable matching algorithm for physical layer security with multiple sourcedestination pairs and jammer nodes," in Proc. 2012 IEEE Wireless Communications and Networking Conference, pp. 2688-2693.

[31] P. Robertson and T. Wörz, "Bandwidth-efficient turbo trellis-coded modulation using punctured component codes," IEEE J. Sel. Areas Commun., vol. 16, pp. 206-218, Feb. 1998.

[32] L. Hanzo, S. X. Ng, T. Keller, and W. Webb, Quadrature Amplitude Modulation: From Basics to Adaptive Trellis-Coded, Turbo-Equalised and Space-Time Coded OFDM, CDMA and MC-CDMA Systems. WileyIEEE Press, 2004.

[33] S. X. Ng, O. R. Alamri, Y. Li, J. K. liewer, and L. Hanzo, "Near-capacity turbo trellis coded modulation design based on EXIT charts and union bounds," IEEE Trans. Commun., vol. 56, no. 12, pp. 2030-2039, 2008.

[34] O. Oyman, N. Laneman, and S. Sandhu, "Multihop relaying for broadband wireless mesh networks: from theory to practice," IEEE Commun. Mag., vol. 45, pp. 116-122, Nov. 2007.

[35] O. Simeone, Y. Bar-Ness, and U. Spagnolini, "Stable throughput of cognitive radios with and without relaying capability," IEEE Trans. Commun., vol. 55, no. 12, pp. 2351-2360, 2007.

[36] H. Vu and H.-Y. Kong, "Joint subcarrier matching and power allocation in OFDM two-way relay systems," J. Commun. and Networks, vol. 14, pp. 257-266, June 2012 .

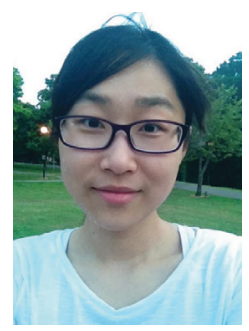

Wei Liang received her B.Eng. degree with First class in electronics engineering from University of Central Lancashire, Lancashire, UK in 2009. She received her M.Sc. degree in wireless communication from University of Southampton, Southampton, U.K in 2010. She is currently working toward the Ph.D. degree in the Communications, Signal Processing and Control Group at the School of Electronics and Computer Science, University of Southampton, Southampton, UK. Her current research interests include adaptive coded modulation, cooperative communication and cognitive radio network.

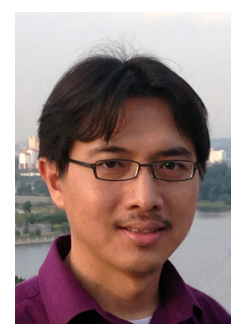

Dr Soon Xin Ng (S'99-M'03-SM'08) received the B.Eng. degree (First class) in electronics engineering and the $\mathrm{Ph} . \mathrm{D}$. degree in wireless communications from the University of Southampton, Southampton, U.K., in 1999 and 2002, respectively. From 2003 to 2006, he was a postdoctoral research fellow working on collaborative European research projects known as SCOUT, NEWCOM and PHOENIX. Since August 2006, he has been a member of academic staff in the School of Electronics and Computer Science, University of Southampton. He is involved in the OPTIMIX and CONCERTO European projects as well as the IU-ATC and UC4G projects. He is currently a senior lecturer at the University of Southampton.

His research interests include adaptive coded modulation, coded modulation, channel coding, space-time coding, joint source and channel coding, iterative detection, OFDM, MIMO, cooperative communications, distributed coding, quantum error correction codes and joint wireless-and-optical-fiber communications. He has published over 160 papers and co-authored two John Wiley/IEEE Press books in this field. He is a Senior Member of the IEEE, a Chartered Engineer and a Fellow of the Higher Education Academy in the UK.

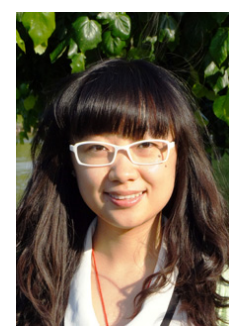

Jiao Feng received her M.Sc. degree in communications engineering from Jilin University, Jilin, China, in 2009 and her Ph.D. degree in wireless communications from the University of Southampton, Southampton, U.K., in 2014 under the support of the China-UK excellence scholarship from both the University of Southampton and the China Scholarship Council. Her research interests include cooperative communication, MAC layer protocols, resource allocation and cognitive radio network. 


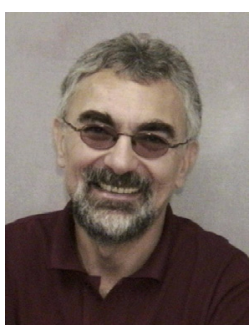

Lajos Hanzo (http://www-mobile.ecs.soton.ac.uk) FREng, FIEEE, FIET, Fellow of EURASIP, DSc received his degree in electronics in 1976 and his doctorate in 1983. In 2009 he was awarded the honorary doctorate "Doctor Honoris Causa" by the Technical University of Budapest. During his 37-year career in telecommunications he has held various research and academic posts in Hungary, Germany and the UK. Since 1986 he has been with the School of Electronics and Computer Science, University of Southampton, UK, where he holds the chair in telecommunications. He has successfully supervised $80+\mathrm{PhD}$ students, co-authored 20 John Wiley/IEEE Press books on mobile radio communications totalling in excess of 10000 pages, published $1400+$ research entries at IEEE Xplore, acted both as TPC and General Chair of IEEE conferences, presented keynote lectures and has been awarded a number of distinctions. Currently he is directing a 100-strong academic research team, working on a range of research projects in the field of wireless multimedia communications sponsored by industry, the Engineering and Physical Sciences Research Council (EPSRC) UK, the European Research Council's Advanced Fellow Grant and the Royal Society's Wolfson Research Merit Award. He is an enthusiastic supporter of industrial and academic liaison and he offers a range of industrial courses. He is also a Governor of the IEEE VTS. During 2008 - 2012 he was the Editor-in-Chief of the IEEE Press and a Chaired Professor also at Tsinghua University, Beijing. His research is funded by the European Research Council's Senior Research Fellow Grant. For further information on research in progress and associated publications please refer to http://www-mobile.ecs.soton.ac.uk Lajos has 19 000+ citations. 